\title{
Link Layer Coding for DVB-S2 Interactive Satellite Services to Trains
}

\author{
Ho-Jin Lee', Pansoo Kim¹, Balazs Matuz², Gianluigi Liva², \\ Cristina Parraga Niebla², Nuria Riera Diaz ${ }^{2}$ and Sandro Scalise ${ }^{2}$ \\ ${ }^{1}$ Broadcasting and communication convergence research division, ETRI, \\ 161 Gajeong-dong Yuseong-gu Daejeon, \\ 2Institute of Communications and Navigation, \\ German Aerospace Center (DLR), 82234 Wessling, \\ ${ }^{1}$ Republic of Korea \\ ${ }^{2}$ Germany
}

\section{Introduction}

The growing number of railway passengers represents an appealing market for multimedia services. Satellites could be used to fulfill these demands due to the large coverage area and the low cost of associated terrestrial infrastructure. However, transmissions to mobile users through satellite links always pose a big challenge, especially since line of sight connection is frequently interrupted by obstacles between the satellite and the mobile receiver. The railroad satellite channel (RSC) in particular suffers from severe fadings that can be described using a combined statistical/deterministic model. In this paper, we will focus on the DVB-S2 [1] forward link providing service to high-speed trains. Additional protection of the data on link layer (LL) has been taken into account to mitigate the fading effects. The LL coding scheme investigated in this paper is based on the adoption of an erasure correcting code whose symbols are packets of constant size. Examples of erasure correcting codes applied in satellite communication systems can be found in [2]-[4]. The effort for the LL code design is mainly focused on the mitigation of the fade events due electrical trellises or power arches (PA) that are placed aside the tracks in order to provide the electric power to the trains along many railways. Such events are frequent and nearly periodic. In [5] it has already been shown that without a proper mitigation technique they would lead to an unacceptable quality of service. The rest of the paper is organized as follows. In Section II. we will provide an overview of the railroad satellite channel, focusing on the effect of electrical trellises on the received signal power level. In Section III the overall system architecture is described. Some insights on the link layer code design are provided as well. Section IV shows a performance comparison between the proposed link-layer coding approach and an enhancement of the DVB-S2 physical layer (PHY layer) through a long inter-frame interleaver. Moreover, a further, simplified model for the railroad satellite channel is introduced to give a basic understanding of the performance for the different solutions. Concluding remarks follow in Section V. 


\section{Railroad satellite channel model}

An appropriate model for the propagation channel in a railway environment can be derived using the land mobile satellite channel (LMSC) as a reference scenario [6]: in the first instance it is sufficient to characterize the channel behavior by two different states, i.e. a line of sight (LOS) state with relatively high received signal power and a non line of sight (NLOS) state where the signal is shadowed or blocked by objects in the vicinity of the receiver. In the former state, the received signal is composed of a direct and a multipath component, with the instantaneous received signal power $S$ obeying a Ricean probability density function:

$$
p_{\text {Rice }}(S)=c \cdot \exp (-c(S+1)) \cdot I_{0}(2 c \sqrt{S}) .
$$

Here, $c$ denotes the so-called Rice factor, i.e. the direct-tomultipath signal power ratio and $I_{0}$ is the modified Bessel function of order zero. In the NLOS state, with no direct signal path present, the signal power shows Rayleigh behavior around a short-term mean value $S_{0}$ with the PDF described by:

$$
p_{\text {Rayl }}\left(S \mid S_{0}\right)=\frac{1}{S_{0}} \exp \left(-S / S_{0}\right)
$$

For the short-term mean $S_{0}$ a lognormal distribution is assumed:

$$
p_{L N}\left(S_{0}\right)=\frac{10}{\sqrt{2 \pi} \sigma_{d B} \ln 10} \cdot \frac{1}{S_{0}} \exp \left[-\frac{\left(10 \log S_{0}-\mu_{d B}\right)^{2}}{2 \sigma_{d B}^{2}}\right],
$$

with $\mu_{d B}$ describing the average power level (in $\mathrm{dB}$ ) and $\sigma_{d B}$ the variance of the power level (in $d B^{2}$ ) due to large scale fading. The railroad satellite channel has some peculiarities that have not been modeled properly by the previous description. Measurement campaigns show that a constant attenuation of 2-3 $\mathrm{dB}$ is introduced by catenaries above the tracks. Also, long fades occur mainly due to structures like bridges or tunnels, and shorter but periodic ones that are caused by several metallic obstacles along the railroad. Among them there are posts (with or without brackets), electrical trellises or arches spanning over the tracks, but they will be simply referred to as power arches for the rest of this paper. In the sequel, we will restrict ourselves on a RSC model corresponding to the LMSC in the LOS case superimposed by short deep fades ascribed to the power arches. Since these fades are nearly-periodic (and thus deterministic), they are not suitable for a statistical characterization. In turn, the modeling approach proposed in [5], here recalled for sake of clarity, will be adopted. The attenuation introduced by the above-mentioned power arches can be accurately described using the knife-edge diffraction theory. The knife-edge attenuation describes the ratio between the received electro-magnetic field $E_{D}$ in presence of an obstacle and the received field under free space conditions $E_{0}$. For an object of two finite dimensions, it can be represented as sum of two diffracted signal components: 


$$
\frac{E_{D}}{E_{0}}=\frac{1+j}{2}\left(\frac{G\left(\alpha_{1}\right)}{G_{M A X}} \int_{v}^{\infty} e^{-j \frac{\pi}{2} t^{2}} d t+\frac{G\left(\alpha_{2}\right)}{G_{M A X}} \int_{-\infty}^{v-\frac{d \cdot v}{h}} e^{-j \frac{\pi}{2} t^{2}} d t\right),
$$

where $d$ is the width of the obstacle, $h$ the height above LOS and $G(\alpha) / G_{\text {MAX }}$ denotes the radiation pattern of the directive antenna. Moreover, the Fresnel parameter $v$ can be calculated out of $h$, the wavelength $\lambda$ and the distance $d_{1}$ between the receiver and the object, as well as the distance $d_{2}$ between the object and the satellite according to:

$$
v=h \sqrt{\frac{2\left(d_{1}+d_{2}\right)}{\lambda d_{1} d_{2}}} .
$$

Following that, the railroad satellite channel which is the basis for all further investigations looks like depicted in Figure 1. It comprises Ricean fading (with a Rice factor of $18 \mathrm{~dB}$ ), as well as periodic deep fades as a result of equally spaced power arches aside the railway. The worse scenario, where additional NLOS is present according to the LMSC model, will not be considered in the sequel.

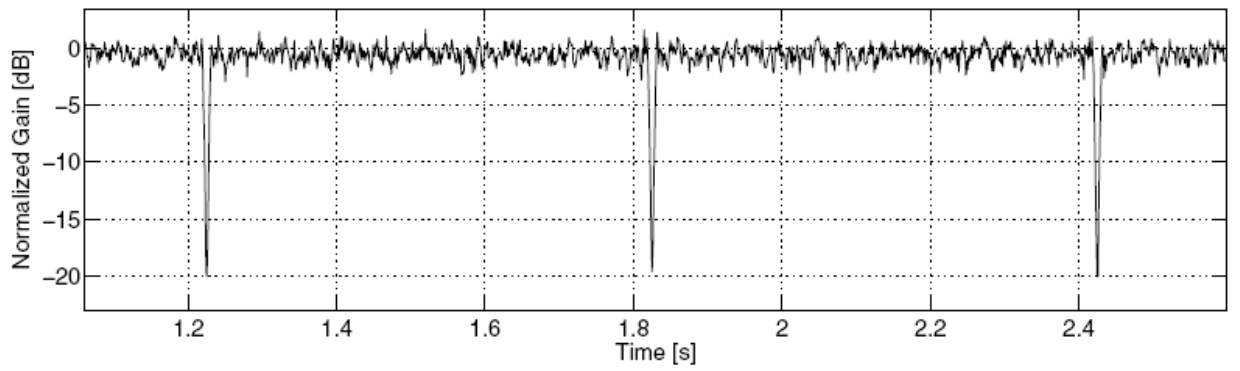

Fig. 1. RSC realization with a power arch distance of $50 \mathrm{~m}$ at a speed of $300 \mathrm{~km} / \mathrm{h}$; periodic deep fades occur every time the train passes by a PA

\section{System description}

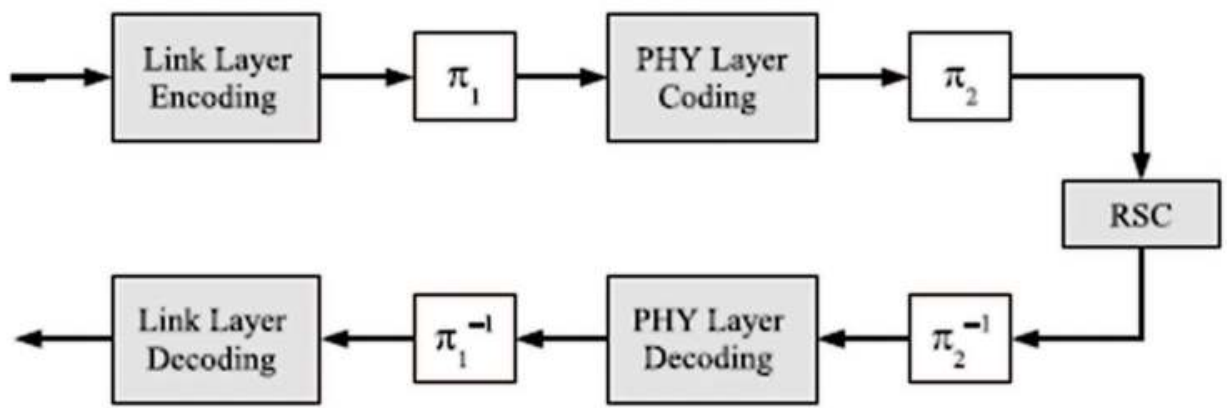

Fig. 2. Transmission chain used for the simulation 
Our approach to mitigate the impairments of the RSC is based on link layer coding. For illustration purposes, a simplified block diagram of the link is depicted in Figure 2. In our case LL coding is applied on MPEG-TS packets. The systematic link layer encoder receives at its input K MPEGTS packets and produces at its output N packets, referred to as LL codeword. Due to the systematic nature of the code, this codeword consist of the K input MPEG-TS packets followed by $\mathrm{M}=\mathrm{N}-\mathrm{K}$ parity packets. On the receiver side, the decoder takes care of recovering lost MPEG-TS packets. Note that in the link layer coding framework the code works on an erasure channel, where the erased units (i.e., the LL codeword symbols) are whole packets. In the context of this paper, such feature is guaranteed by the error detection performed after physical layer decoding on each MPEGTS/parity packet. In other words, the PHY decoder attempts to protect individual MPEG-TS packets, whereas the LL decoder is meant to recover lost MPEG-TS packets. The LL decoder design is highly facilitated by the underling erasure channel, permitting for some kind of codes the adoption of software based decoding up to several tens of Mbytes per second. To allow this appealing feature, our investigation is focused on the adoption of low-density parity-check (LDPC) codes [7] as erasure correcting codes. LDPC codes provide capacity approaching performance on many communication channels [8], and in the framework of LDPC codes some astonishing erasure correcting codes have been developed [9]-[11]. The LDPC codes adopted for the simulations belong to the family of the so-called irregular repeat-accumulate (IRA) codes [12]. IRA codes allow simple efficient encoding while keeping nearcapacity performance. The design of the IRA codes have been optimized through extrinsic information transfer (EXIT) analysis [13]-[15], constraining the parity-check matrix of the code to a block-circulant form that would also permit a simple hardware decoder design. Further performance enhancements with similar encoding complexities are expected by adopting more sophisticated IRA-like designs [16] [17]. After link layer coding is done, the MPEG-TS packets within each LL codeword are interleaved (denoted by $\pi_{1}$ in Figure 2) to break up channel correlations. For our investigations we limited the maximum length of the link layer codeword in a way that it spans over to $200 \mathrm{~ms}$. For example, taking into account a symbol rate of 27.5 MBaud, QPSK modulation and physical layer code rate $r=1 / 2$, the LL codeword length would be $\mathrm{N}=3400$ packets. This constraint has been introduced to avoid long delays which could affect real-time applications. The stream of MPEG-TS/parity packets is then forwarded to a DVB-S2 transmitter, which takes care of the physical layer coding through the serial concatenation of a BCH (Bose, Ray-Chaudhuri, Hocquenghem) code and LDPC code according to the DVB-S2 standard (for details see [1]). The physical layer codeword size corresponds to the large frame size of the DVB-S2 standard (64800 bits). Before forwarding the data to radio frequency (RF) frontend, we allow a further (optional) physical layer inter-frame block interleaver $\pi_{2}$ that permutes the bits among several frames. For the sake of comparison, in the following we will consider also the scenario where the LL coding block is disabled. In such case, the diversity necessary to overcome the short periodic fade events will be provided by the inter-frame interleaver only. However, the interleaver latency will be constrained to be lower than $200 \mathrm{~ms}$. Furthermore, to keep the comparison fair, physical layer code rate will be lowered in a way that the overall efficiency of the two systems is the same. 


\section{Outcomes}

In section IV, we provide some numerical results obtained through Monte Carlo simulations on the RSC described in Section II. The analysis is focused on the case of LOS conditions with superimposed power arches. The performance is depicted in terms of MPEG-TS packet error rate (PER) versus signal-to-noise ratio (SNR) $E_{s} / N_{0}$. Here, $E_{s}$ denotes the energy per modulated symbol and $N_{0}$ the one-sided noise power spectral density. For the LL coded solution, the results are shown in terms of residual MPEG-TS packet error rate at the output of the LL decoder vs. $E_{s} / N_{0}$. Besides the PA width and the train velocity that have a high impact on the fade duration, also other factors have to be taken into account for the simulations, such as the current latitude and the traveling direction of the train. To simplify the simulations it is advisable to determine an effective power arch width that takes into account these factors. For a PA width of $30 \mathrm{~cm}$ and a latitude of $38^{\circ}$ geometric considerations yield to an effective PA width of roughly $87 \mathrm{~cm}$. Considering train speeds from $30 \mathrm{~km} / \mathrm{h}$ to $150 \mathrm{~km} / \mathrm{h}$ and a north-south traveling direction, the resulting fade durations range from $\sim 100 \mathrm{~ms}$ to $\sim 20 \mathrm{~ms}$. The distance between two subsequent PA is constantly set to $50 \mathrm{~m}$.

\subsection{Simulation results}

Assuming only PHY layer coding with a code rate of $1 / 4$ combined with physical layer interleavers of different lengths we obtain the plots in Figure 3 (at $30 \mathrm{~km} / \mathrm{h}$ on the left, at 150 $\mathrm{km} / \mathrm{h}$ on the right). For both speeds, intermediate error floors arise at error rates proportional to the interleaver duration (in the charts, the performance with interleaver lengths of $200 \mathrm{~ms}, 100 \mathrm{~ms}, 50 \mathrm{~ms}$ and no interleaving are depicted). However, at high speeds the rate $1 / 4$ code in combination with a sufficiently long interleaver is able to overcome the floor, but the steepness of the curve in the subsequent waterfall region remains quite poor. For low speeds the error floors remain, for all the investigated interleaver lengths and also for relatively high SNRs. The outcomes for joint physical/link layer coding with rate 1/2 codes on both layers and with a LL interleaver duration of $200 \mathrm{~ms}$ combined with different PHY layer interleaver durations are shown in Figure 4 (at $30 \mathrm{~km} / \mathrm{h}$ on the left, at $150 \mathrm{~km} / \mathrm{h}$
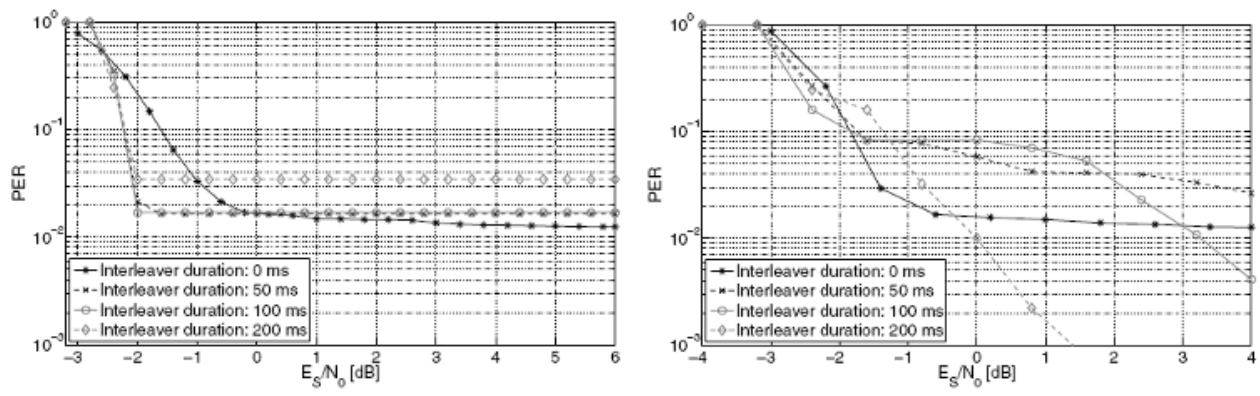

Fig. 3. PER vs Es/No with only physical layer protection(DVB-S2, QPSK, $r=1 / 4$ ) and different interleaving depths. Overall spectral efficiency of $0.5 \mathrm{bps} / \mathrm{Hz}$. Speed of $30 \mathrm{~km} / \mathrm{h}$ (left) and $150 \mathrm{~km} / \mathrm{h}$ (right) 
on the right). The best results can be achieved by using no physical interleaver at all. In this case the LL code is able to overcome the errorfloor at both speeds and ensures a steep slope of the PER curve in the waterfall region. Compared to plain physical layer coding with inter-frame interleaving, joint physical/link layer coding clearly shows an improvement of performance. Note that for joint PHY/LL coding both interleavers $\pi_{1}$ and $\pi_{2}$ are synchronized, so that the overall delay that is experienced due to interleaving is equivalent to the maximum of the delays introduced by $\pi_{1}$ and $\pi_{2}$ (in our case not more than $200 \mathrm{~ms})$.
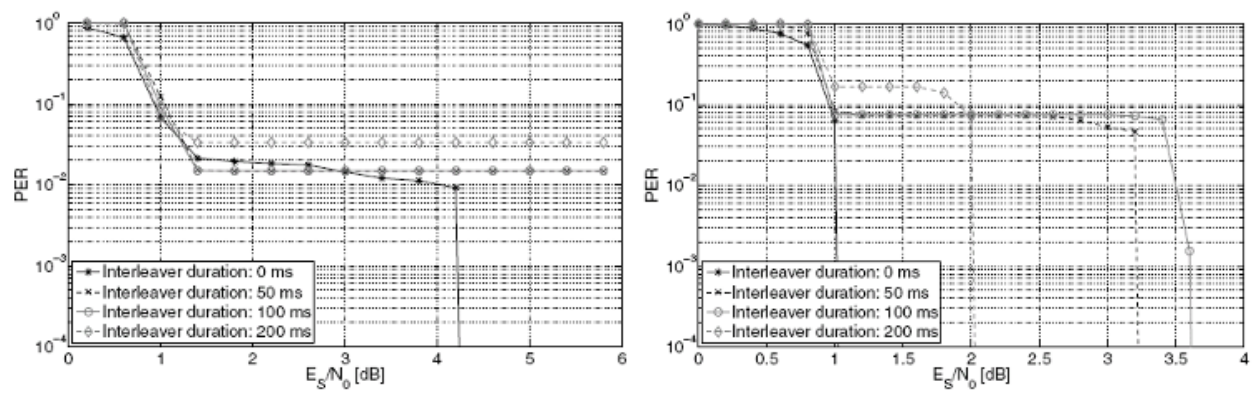

Fig. 4. PER vs Es/No with joint physical layer protection(DVB-S2, QPSK, r=1/2) and link layer Protections(link layer LDPC code, $R=1 / 2$ ) and different interleaving depths. Speed of $30 \mathrm{~km} / \mathrm{h}$ (left) and $150 \mathrm{~km} / \mathrm{h}$ (right). Overall spectral efficiency of $0.5 \mathrm{bps} / \mathrm{Hz}$

\subsection{Insights on the use of PHY layer interleavers on the RSC}

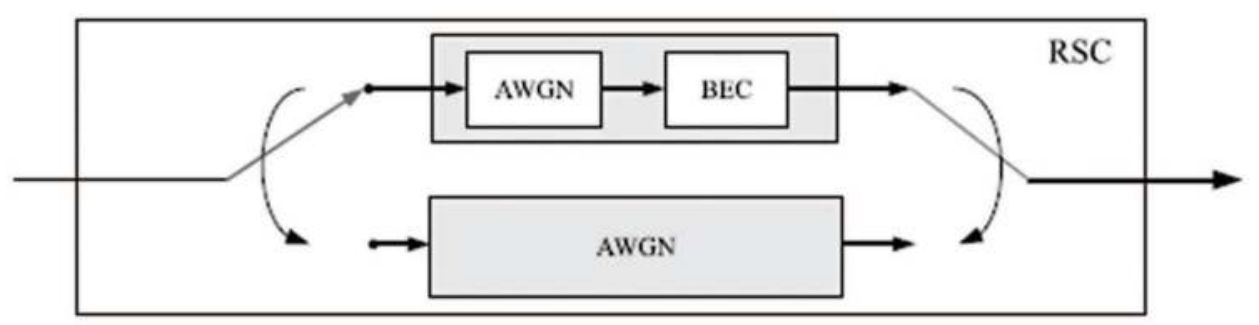

Fig. 5. Main concept of the AWGN/EC-AWGN channel model for the RSC channel

The behavior of a coding scheme including physical layer coding and long inter-frame interleavers on the LOS channel with superimposed blockages can be easily understood by splitting the contributions to the packet error probability $P_{e}$ (that is the stochastic equivalent to the simulated PER) into two parts, the LOS error rate and the blockage error rate. The LOS condition is referred as the good state $(G)$. To simplify the analysis in such state, the channel characteristic is approximated by an AWGN channel (recall the high Rice factor in LOS). The blockage condition ascribed to PAs is referred to as the bad state $(B)$. Here, the channel is basically a bursty erasure channel. Assuming interleaving windows longer than a 
PA fade duration (thus, spreading the erasures on a wider duration), the PHY layer decoder deals with a combination of an erasure channel with AWGN (EC-AWGN). This state spans over a whole interleaver window. As an illustration the overall channel model is depicted in Figure 5. Denoting by $\mathrm{X}$ the channel state random variable, the stationary probabilities of being in the good/bad state are given by

$$
P_{G}=\frac{T_{G}}{T_{B}+T_{G}} \text { and } P_{B}=\frac{T_{B}}{T_{B}+T_{G}}
$$

where $T_{G}\left(T_{B}\right)$ represents the time spent in the good (bad) state. Assuming periodic fade events due to the power arches, $T_{B}$ shall be replaced by the interleaver length $L$, expressed in seconds, or by the fade duration $T_{f}$, in case no interleaving is applied. For sake of simplicity, let's summarize such parameter as $\Delta_{B}$. The sum $T_{B}+T_{G}$ has to be replaced by the power arch periodicity $\tau$, while $T_{G}$ becomes the time interval between two interleaving windows affected by consecutive power arch fades, $\Delta_{G}$. Let's define $\operatorname{Pr}\{E \mid X=G\}$ and $\operatorname{Pr}\{E \mid X=B\}$ as the packet error conditional probabilities given the good (bad) state, where $E$ denotes the packet error event. The error probability $P_{e}$ can be therefore expressed as

$$
\begin{aligned}
P_{e} & =P_{G} \cdot \operatorname{Pr}\{E \mid X=G\}+P_{B} \cdot \operatorname{Pr}\{E \mid X=B\} \\
& =\frac{\Delta_{G}}{\Delta_{G}+\Delta_{B}} \operatorname{Pr}\{E \mid X=G\}+\frac{\Delta_{B}}{\Delta_{G}+\Delta_{B}} \operatorname{Pr}\{E \mid X=B\} .
\end{aligned}
$$

Note that, due to the parameters chosen for the simulations, $\Delta_{B} \sim 10^{-2} \cdot \Delta_{G}$. Consequently, $P_{B}$ is in the order of magnitude of $10^{-2}$. Thus, at low SNRs, where the error rates are high even in LOS conditions, the first term in (1) dominates the summation. At high SNRs, the error rate $\operatorname{Pr}\{E \mid X=G\}$ in LOS condition quickly decreases. The second term becomes therefore dominant. The performance curve of the system in such conditions can be composed in a two-fold fashion:

1. The error probability $\operatorname{Pr}\{E \mid X=G\}$ in LOS conditions is evaluated numerically down to error rates that are negligible respect to $P_{B}$. With the current scenario of $P_{B} \sim 10^{-2}$ the simulation can be stopped once $\operatorname{Pr}\{E \mid X=G\}$ approaches $10^{-3}$

2. As a second step the probability $\operatorname{Pr}\{E \mid X=B\}$ for the EC-AWGN channel has to be computed. In case no interleaving is applied, $\Delta_{B}$ is equal to $T_{f}$. In this interval $\operatorname{Pr}\{E \mid X=G\}$ can be reasonably set to 1 . Otherwise, the problem of computing $\operatorname{Pr}\{E \mid X=B\}$ reduces to the performance evaluation of the channel code on the ECAWGN, where, assuming random interleaving, each bit soft-value is erased with a probability $\varepsilon$, with $\varepsilon=T_{f} / L$ in the interval $\Delta_{B}=L$.

The two error probabilities are then combined on the same chart following equation (1). This is exemplified in Figure 6. The LL code employed for the simulation is a short $(2048,1024)$ LDPC code. The impact of the physical layer interleaver length becomes therefore quite clear: large interleavers lower the erasure rate (recall that $\varepsilon=T_{f} / L$ ) of the codeword bits in blockage conditions, increasing the steepness of the $\operatorname{Pr}\{E \mid X=B\}$ curve. At the same time, high values of $L$ rise up the intermediate floor, at the error rate given by $P_{B}=L / \tau$. This is 
compliance with the results presented in charts 3 and 4: the higher the interleaver length, the higher the intermediate error floor. As it can be seen, there are some exceptions, since the same error floor arises for the $50 \mathrm{~ms}$ and $100 \mathrm{~ms}$ interleavers. This is due to the fact that PA fades affect two interleaver windows for the $50 \mathrm{~ms}$ case (i.e., the interleaving window has a length which comparable to the fade duration). In case of a scheme employing a link level code on top of the physical layer, it is often advisable to abstain from the use of physical layer interleavers to keep the intermediate error floors as low as possible (especially at low train speeds). The packet recovery task in presence of the PA fades is then left to the LL code.

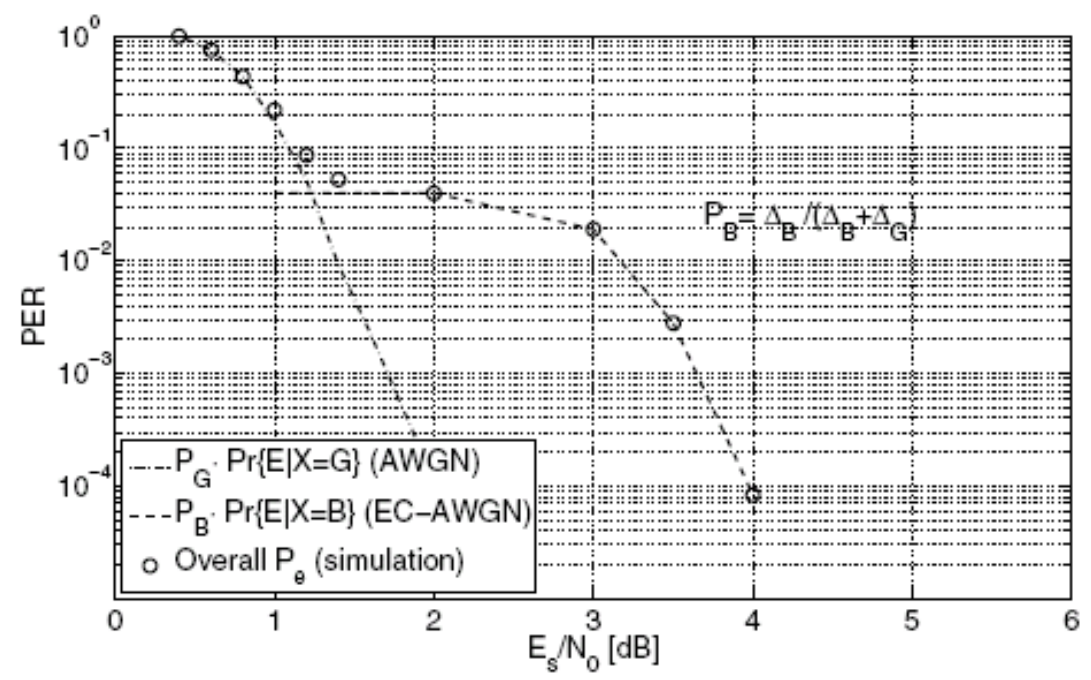

Fig. 6. Packet error rate for the simplified RSC (hybrid AWGN/EC-AWGN model)

\section{Concluding remarks}

The main purpose of this work was to draw a comparison between physical layer coding and interleaving and the innovative approach of joint physical/link layer coding for the railroad satellite channel. It was shown that the system performance can be highly improved for this type of channel by splitting redundancy on different layers. Employing link layer coding shows some performance advantages with respect to the use of long physical layer interleavers, especially in case of frequent short blockages. A simplified model for the LOS railroad satellite channel with superimposed periodic fades was introduced, with focus on the performance of a scheme employing physical layer coding enhanced by long inter-frame interleavers. The proposed model allows a precise calculation of the arising error floors, as well as simple explanation of the system behavior for different interleaver lengths and train velocities. This knowledge turns out to be very helpful for the code design. 


\section{Acknowledgments}

This work was supported by the ETRI-DLR Collaborative Research under the name of "Communication Technologies for Satellite Broadband Mobile based on DVB-S2/RCS". The work has been presented in part at VTC 2008 spring[18].

\section{References}

[1] Digital Video Broadcasting (DVB): Second generation framing structure,channel coding and modulation systems for Broadcasting, Interactive Services, News Gathering and other broadband satellite applications, ETSI Std. EN 302 307, 2004.

[2] C. Di, H. Ernst, E. Paolini, S. Coletto, and M. Chiani, Low-density parity-check codes for the transport layer of satellite broadcast, in Proc. AIAA International Communications Satellite Systems Conference(ICSSC 2005), Rome, Italy, Sep. 2005.

[3] M. Chiani, G. Liva, and E. Paolini, Investigation of long erasure codes for space communication protocols, CCSDS, Rome, Tech. Rep., June 2006, spring Meeting.

[4] E. Paolini, G. Liva, M. Chiani, and G. Calzolari, Tornado-like codes: a new appealing chance for space applications protocols?, in 3rd European Space Agency Workshop on Tracking, Telemetry and Command Systems for Space Applications, TTC 2004, Sep. 2004.

[5] S. Scalise, R. Mura, and V. Mignone, Air Interfaces for Satellite Based Digital TV Broadcasting in the Railway Environment, in IEEE Transactions on Broadcasting, IEEE, Ed., vol. 52, no. 2, June 2006, pp. 158-166.

[6] E. Lutz, M. Werner, and A. Jahn, Satellite Systems for Personal andBroadband Communications. Springer Verlag, 2000.

[7] R. G. Gallager, Low-Density Parity-Check Codes. Cambridge, MA: M.I.T. Press, 1963.

[8] T. Richardson and R. Urbanke, The capacity of low-density parity check codes under message-passing decoding, IEEE Trans. Inform Theory, vol. 47, 2001.

[9] M. Luby, LT-codes, in Proc. of the ACM Symposium on Foundations of Computer Science (FOCS), 2002.

[10] E. Paolini, M. Fossorier, and M. Chiani, Analysis of doubly-generalized LDPC codes with random component codes for the binary erasure channel, in Proc. of Allerton Conf. on Communications, Control and Computing, Monticello, USA, Sep. 2006.

[11] A. Shokrollahi, Raptor codes, IEEE Transactions on Information Theory, vol. 52, no. 6, pp. 2551-2567, Jun. 2006.

[12] H. Jin, A. Khandekar, and R. McEliece, Irregular repeat-accumulate codes, in Proc. International Symposium on Turbo codes and Related Topics, Sep. 2000, pp. 1-8.

[13] S. ten Brink, Convergence behavior of iteratively decoded parallel concatenated codes, IEEE Trans. Commun., vol. 49, pp. 1727-1737, Oct. 2001.

[14] G. Liva and M. Chiani, Protograph LDPC codes design based on EXIT analysis, in Proc. IEEE Globecomm, Nov. 2007.

[15] G. Liva, S. Song, L. Lan, Y. Zhang, W. Ryan, and S. Lin, Design of LDPC codes: A survey and new results, J. Comm. Software and Systems, Sep. 2006. 
[16] A. Abbasfar, K. Yao, and D. Disvalar, Accumulate repeat accumulate codes, in Proc. IEEE Globecomm, Dallas, Texas, Nov. 2004.

[17] G. Liva, E. Paolini, and M. Chiani, Simple reconfigurable low-density parity-check codes, IEEE Comm.Letters, vol. 9, pp. 258-260, March 2005.

[18] B. Matus, Link Layer Coding for DVB-S2 Interactive Satellite Services to Trains, in Proc. IEEE VTC, Sigapore, May. 2008 


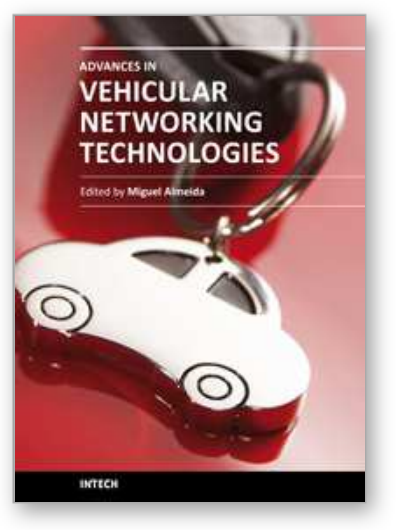

\author{
Advances in Vehicular Networking Technologies \\ Edited by Dr Miguel Almeida
}

ISBN 978-953-307-241-8

Hard cover, 432 pages

Publisher InTech

Published online 11, April, 2011

Published in print edition April, 2011

This book provides an insight on both the challenges and the technological solutions of several approaches, which allow connecting vehicles between each other and with the network. It underlines the trends on networking capabilities and their issues, further focusing on the MAC and Physical layer challenges. Ranging from the advances on radio access technologies to intelligent mechanisms deployed to enhance cooperative communications, cognitive radio and multiple antenna systems have been given particular highlight.

\title{
How to reference
}

In order to correctly reference this scholarly work, feel free to copy and paste the following:

Ho-Jin Lee, Pansoo Kim, Balazs Matuz, Gianluigi Liva, Cristina Parraga Niebla, Nuria Riera Dıaz and Sandro Scalise (2011). Link Layer Coding for DVB-S2 Interactive Satellite Services to Trains, Advances in Vehicular Networking Technologies, Dr Miguel Almeida (Ed.), ISBN: 978-953-307-241-8, InTech, Available from: http://www.intechopen.com/books/advances-in-vehicular-networking-technologies/link-layer-coding-for-dvb-s2interactive-satellite-services-to-trains

\section{INTECH}

open science | open minds

\section{InTech Europe}

University Campus STeP Ri

Slavka Krautzeka 83/A

51000 Rijeka, Croatia

Phone: +385 (51) 770447

Fax: +385 (51) 686166

www.intechopen.com

\section{InTech China}

Unit 405, Office Block, Hotel Equatorial Shanghai

No.65, Yan An Road (West), Shanghai, 200040, China

中国上海市延安西路 65 号上海国际贵都大饭店办公楼 405 单元

Phone: +86-21-62489820

Fax: +86-21-62489821 
(C) 2011 The Author(s). Licensee IntechOpen. This chapter is distributed under the terms of the Creative Commons Attribution-NonCommercialShareAlike-3.0 License, which permits use, distribution and reproduction for non-commercial purposes, provided the original is properly cited and derivative works building on this content are distributed under the same license. 